Pacific

Journal of

Mathematics

CONSTRUCTION OF ELLIPTIC CURVES WITH NONINTEGER TORSION POINTS AND NONCYCLIC TORSION GROUPS

Sumi JeONG AND HWASIN PARK

Volume 244 No. 2

February 2010 


\title{
CONSTRUCTION OF ELLIPTIC CURVES WITH NONINTEGER TORSION POINTS AND NONCYCLIC TORSION GROUPS
}

\author{
Sumi JEONG AND HWASIN PARK
}

We study elliptic curves in Weierstrass form (with integer coefficients) that have noninteger torsion points over $\mathbb{Q}$. After putting the curves in certain normal forms, we find conditions on their coefficients characterizing when the torsion group is isomorphic to $\mathbb{Z} / 2 \mathbb{Z} \times \mathbb{Z} / 2 N \mathbb{Z}$, for each $N=1,2,3,4$.

\section{Introduction and main results}

Let $E$ be an elliptic curve given by the Weierstrass equation

$$
y^{2}+a_{1} x y+a_{3} y=x^{3}+a_{2} x^{2}+a_{4} x+a_{6}, \quad a_{i} \in \mathbb{Z},
$$

and assume $E$ has a noninteger torsion point in the projective plane $\mathbb{P}^{2}(\mathbb{Q})$. Then $E$ has one of the following expressions, up to a translation $(x, y) \mapsto(x-\alpha, y-\beta)$ with $\alpha, \beta \in \mathbb{Z}$ :

$$
\begin{aligned}
& E_{1}: y^{2}+x y=x^{3}+4(a-4 b) x^{2}+a x+b, \\
& E_{2}: y^{2}+x y+y=x^{3}+2(2 a-8 b-1) x^{2}+a x+b .
\end{aligned}
$$

(See Theorem 1.) Here $a, b \in \mathbb{Z}$. The curve $E_{1}$ has a noninteger torsion point at $\left(-\frac{1}{4}, \frac{1}{8}\right)$, and $E_{2}$ one at $\left(-\frac{1}{4},-\frac{3}{8}\right)$.

If a curve of the form $E_{1}$ or $E_{2}$ has a noninteger point in $\mathbb{P}^{2}(\mathbb{Q})$ apart from $\left(-\frac{1}{4}, \frac{1}{8}\right)$ or $\left(-\frac{1}{4},-\frac{3}{8}\right)$, respectively, that point has infinite order, so the curve has rank at least 1. In Section 2 we give explicit examples.

Since $E_{1}$ and $E_{2}$ have a 2-torsion point, we may ask for what choices of the coefficients the torsion subgroup is isomorphic to $\mathbb{Z} / 2 \mathbb{Z} \times \mathbb{Z} / 2 N \mathbb{Z}$, for $N=1,2,3$, or 4 (higher values of $N$ being excluded by [Mazur 1977]). In Sections 3 and 4, we give criteria for determining whether a curve of the form $E_{1}$ or $E_{2}$ has such a noncyclic torsion subgroup, and construct all possible families of elliptic curves with noninteger torsion points and a noncyclic torsion subgroup. Specifically:

MSC2000: 11G05, 14G05.

Keywords: elliptic curve. 
- Let $m, n$ be integers of the same parity and set

$$
E_{11}: y^{2}+x y=x^{3}+2(m+n) x^{2}+\left(4 m n+\frac{1}{2}(m+n)\right) x+m n
$$

as a particular case of $E_{1}$. The torsion group of such curves contains a $(\mathbb{Z} / 2 \mathbb{Z})^{2}$ (Theorem 8 ). We also construct a similar family based on $E_{2}$ (Theorem 13).

- No curve $E_{2}$ can have torsion subgroup $\mathbb{Z} / 2 \mathbb{Z} \times \mathbb{Z} / 4 \mathbb{Z}$ or $\mathbb{Z} / 2 \mathbb{Z} \times \mathbb{Z} / 8 \mathbb{Z}$ (Theorem $14)$. For the case of $E_{1}$, we construct curves with these torsion groups as follows:

(a) Assume that $k, s$ are integers, with $s>0$. If we choose

$$
m=-k-2 k^{2} \text { and } n=m+2 s^{2},
$$

then $E_{11}$ has a torsion subgroup isomorphic to $\mathbb{Z} / 2 \mathbb{Z} \times \mathbb{Z} / 4 \mathbb{Z}$ (Theorem 10).

(b) Let $(v, w, \square)$ be a primitive Pythagorean triple with $v$ even, and let $t$ be a nonnegative integer. For $s=v^{2}(4 t+1) / 4$ and $k=\left(w^{2}(4 t+1)-1\right) / 4$ or $s=v^{2}(4 t+3) / 4$ and $k=-\left(w^{2}(4 t+3)+1\right) / 4$, the elliptic curve $E_{11}$ defined by (1-2) and (1-3) has torsion group $\mathbb{Z} / 2 \mathbb{Z} \times \mathbb{Z} / 8 \mathbb{Z}$ (Theorem 15 ).

Elliptic curves with torsion subgroup isomorphic to $\mathbb{Z} / 2 \mathbb{Z} \times \mathbb{Z} / 8 \mathbb{Z}$ have been classified (see [Campbell and Goins 2004, Theorem 6.2], for example). They all can be written in the form just given (Remark 16).

- We construct elliptic curves with torsion group $\mathbb{Z} / 2 \mathbb{Z} \times \mathbb{Z} / 6 \mathbb{Z}$ in Theorems 12 (d) and 14(c). Via coordinate transformations these curves take the form

$$
y^{2}=\left(x+\frac{(s+t)^{2}}{4}\right)\left(x+\frac{(s-t)^{2}}{4}\right)\left(x+\frac{(s+t)^{2}(s-t)^{2}}{16 t^{2}}\right)
$$

where $s, t$ are integers satisfying $s \neq t,-3 t, t \mid s^{2}$ and $s \equiv t \equiv 1$ or $3(\bmod 4)$.

\section{Elliptic curves with noninteger torsion points in $\mathbb{P}^{2}(\mathbb{Q})$ and rank $\geq 1$}

Given an elliptic curve in Weierstrass form (1-1), we can replace $y$ by $y-a_{1} x / 2$ if $a_{1}$ is even, or by $y-\left(a_{1}-1\right) x / 2$ if $a_{1}$ is odd, to obtain an isomorphic curve in Weierstrass form with $a_{1}=0$ or $a_{1}=1$.

By the Lutz-Nagell theorem, if $a_{1}=0$ and $P=\left(x_{P}, y_{P}\right)$ is a rational torsion point, then $x_{P}$ and $y_{P}$ are integers. Because we are interested in finding elliptic curves with noninteger torsion points, we therefore restrict our attention to the case $a_{1}=1$; that is, our curve has the form

$$
E^{\prime}: y^{2}+x y+a_{3} y=x^{3}+a_{2} x^{2}+a_{4} x+a_{6}, \text { where } a_{2}, a_{3}, a_{4}, a_{6} \in \mathbb{Z} .
$$

Theorem 1. Assume the curve $E^{\prime}$ in (2-1) has noninteger torsion points. Then, up to a coordinate translation $(x, y) \mapsto(x-\alpha, y-\beta)$ with $\alpha, \beta \in \mathbb{Z}$, the curve is of 
one of the following forms, where $a, b$ are integers:

$$
\begin{aligned}
& E_{1}: y^{2}+x y=x^{3}+4(a-4 b) x^{2}+a x+b, \\
& E_{2}: y^{2}+x y+y=x^{3}+2(2 a-8 b-1) x^{2}+a x+b .
\end{aligned}
$$

Proof. By the Lutz-Nagell theorem, a noninteger torsion point $P$ of $E^{\prime}$ in $\mathbb{P}^{2}(\mathbb{Q})$ must have order two and coordinates $\left(x_{P}, y_{P}\right)=\left(m / 2^{2}, n / 2^{3}\right)$, with $m, n \in \mathbb{Z}$. From $2 P=O$, the group law gives

$$
2 y_{P}+x_{P}+a_{3}=0,
$$

hence $n+m=-4 a_{3}$. This implies $n+m \equiv 0(\bmod 4)$. We thus have the following possibilities for $P$, where $\alpha$ and $\beta$ are integers:

- $m \equiv 0(\bmod 4), n \equiv 0(\bmod 4) \Longrightarrow P=\left(\alpha, \beta+\frac{1}{2}\right)$.

- $m \equiv 1(\bmod 4), n \equiv 3(\bmod 4) \Longrightarrow P=\left(\alpha+\frac{1}{4}, \beta-\frac{1}{8}\right)$ or $P=\left(\alpha+\frac{1}{4}, \beta+\frac{3}{8}\right)$.

- $m \equiv 2(\bmod 4), n \equiv 2(\bmod 4) \Longrightarrow P=\left(\alpha+\frac{1}{2}, \beta \pm \frac{1}{4}\right)$.

- $m \equiv 3(\bmod 4), n \equiv 1(\bmod 4) \Longrightarrow P=\left(\alpha-\frac{1}{4}, \beta+\frac{1}{8}\right)$ or $P=\left(\alpha-\frac{1}{4}, \beta-\frac{3}{8}\right)$.

By a coordinate translation, we can assume $\alpha=0$ and $\beta=0$, so the possibilities for $P$ after this reduction are $\left(0, \frac{1}{2}\right),\left(\frac{1}{2}, \pm \frac{1}{4}\right),\left( \pm \frac{1}{4}, \mp \frac{1}{8}\right)$ and $\left( \pm \frac{1}{4}, \pm \frac{3}{8}\right)$. However, not all them can occur. If $P=\left(\frac{1}{2}, \frac{1}{4}\right)$, for example, the equality $2 y_{P}+x_{P}+a_{3}=0$ gives $a_{3}=-1$, so $\frac{1}{16}+\frac{1}{8}-\frac{1}{4}=\frac{1}{8}+\frac{1}{4} a_{2}+\frac{1}{2} a_{4}+a_{6}$, which is impossible for $a_{2}, a_{4}, a_{6} \in \mathbb{Z}$. A similar calculation excludes all but two cases:

- $P=\left(-\frac{1}{4}, \frac{1}{8}\right) \Longrightarrow a_{3}=0, a_{2}=4 a_{4}-16 a_{6}$,

- $P=\left(-\frac{1}{4},-\frac{3}{8}\right) \Longrightarrow a_{3}=1, a_{2}=4 a_{4}-16 a_{6}-2$.

Setting $a=a_{4}$ and $b=a_{6}$ yields the forms in (2-2).

Remark 2. It follows that, if an elliptic curve of the form $E_{1}$ or $E_{2}$ has a noninteger point in $\mathbb{P}^{2}(\mathbb{Q})$ other than $\left(-\frac{1}{4}, \frac{1}{8}\right)$ or $\left(-\frac{1}{4},-\frac{3}{8}\right)$, respectively, that point contributes to the rank of the curve. Similarly, if a curve is not isomorphic to either $E_{1}$ or $E_{2}$ and has a noninteger rational point, this point contributes to the rank, since it is not a torsion point.

Remark 3. The condition (2-3) in the proof of Theorem 1 being also sufficient for $P$ to have order 2, any curve of the form (2-2) with integer $a, b-$ so long as it is nonsingular - has a point of order 2 with coordinates $\left(-\frac{1}{4}, \frac{1}{8}\right)$ (in the case of $E_{1}$ ) or $\left(-\frac{1}{4},-\frac{3}{8}\right)$ (for $\left.E_{2}\right)$.

Theorem 4. Consider the elliptic curve $E_{1}: y^{2}+x y=x^{3}+4(a-4 b) x^{2}+a x+b$, where $a, b \in \mathbb{Z}$. If $p / q \in \mathbb{Q}$ is the $x$-coordinate of a point in $E_{1}(\mathbb{Q})$, where $p, q$ are relatively prime integers with $q>0$, then $q$ is a square. Further, if $q=4$, then $p \equiv 3(\bmod 4)$. 
Proof. Setting $x=p / q$ in the equation for $E_{1}$, we see $y$ is rational if and only if

$$
q(4 p+q)\left(p^{2}+4 a p q-16 b p q+4 b q^{2}\right) \text { is a square, }
$$

or equivalently, with $q=q^{\prime} r^{2}$, where $r \in \mathbb{Z}$ and $q^{\prime}$ is a square-free positive integer,

$$
q^{\prime}(4 p+q)\left(p^{2}+4 a p q-16 b p q+4 b q^{2}\right) \text { is a square. }
$$

Assume this is the case. Then $q^{\prime} \mid(4 p+q)\left(p^{2}+4 a p q-16 b p q+4 b q^{2}\right)$, which is the same as $q^{\prime} \mid 4 p^{3}$. But $q^{\prime} \nmid p^{3}$, since $\operatorname{gcd}(p, q)=1$, so we get $q^{\prime}=1$ or $q^{\prime}=2$. If $q^{\prime}=2$, then $p$ is odd and $\left(2 p+r^{2}\right)\left(p^{2}+8 a p r^{2}-32 b p r^{2}+16 b r^{4}\right)$ is a square, by $(2-5)$. But this cannot be so, because this expression is congruent $(\bmod 4)$ to $\left(2 p+r^{2}\right) p^{2} \equiv\left(2+r^{2}\right) \cdot 1 \equiv 2$ or 3 . This contradiction shows that $q^{\prime}=1$; that is, $q$ is a square.

If $q=4$, again $p$ is odd and (2-5) implies that $(p+1)\left(p^{2}+16 a p-64 b p+64 b\right)$ is a square. This reduces $(\bmod 4)$ to $(p+1) p^{2} \equiv p+1$. Hence $p \equiv 3(\bmod 4)$.

We can use Remark 2 and Theorem 4 to construct a family of elliptic curves with rank at least 1 .

Example 5. For $k, t \in \mathbb{Z}$, consider the elliptic curve

$$
E: y^{2}+x y=x^{3}-\left(16 k^{2}+12 k+16 t\right) x^{2}+\left(12 k^{2}+9 k+8 t\right) x+\left(4 k^{2}+3 k+3 t\right) \text {. }
$$

$E(\mathbb{Q})$ contains the point $\left(\frac{3}{4}, \frac{1}{8}(3+16 k)\right)$ and its additive inverse $\left(\frac{3}{4}, \frac{1}{8}(-9-16 k)\right)$; being noninteger and distinct from $\left(-\frac{1}{4}, \frac{1}{8}\right)$, these points have infinite order. Hence the curve has rank at least 1 .

To see how the example is obtained, we need only consider the conditions on $a, b \in \mathbb{Z}$ such that $E_{1}$ in Theorem 4 has a rational point $(x, y)$ with $x=\frac{3}{4}$. Substitution gives $y=\frac{1}{8}(-3 \pm 2 \sqrt{9+48 a-128 b})$; that is, we must find conditions on $a, b$ ensuring that $9+48 a-128 b=A^{2}$ for some $A \in \mathbb{Z}$. Since $16(3 a-8 b)=A^{2}-9$, we have $16 \mid\left(A^{2}-9\right)$, or, upon replacing $A$ by $-A$ if necessary, $A \equiv 3(\bmod 8)$. Put $A=8 k+3$ for some $k \in \mathbb{Z}$; then $3 a-8 b=4 k^{2}+3 k$. Since $(3,8)=1$, we can write $a=3\left(4 k^{2}+3 k\right)+8 t$ and $b=\left(4 k^{2}+3 k\right)+3 t$ for some $t \in \mathbb{Z}$. We find the value $8 k+3$ for the radical and hence the values of $y$. Working backwards, or simply checking by substitution, we see that any $k, t \in \mathbb{Z}$ will work.

Theorem 6. Consider the elliptic curve $E_{2}: y^{2}+x y+y=x^{3}+2(2 a-8 b-1) x^{2}+$ $a x+b$, where $a, b \in \mathbb{Z}$. If $p / q \in \mathbb{Q}$ is the $x$-coordinate of a point in $E_{2}(\mathbb{Q})$, where $p, q$ are relatively prime integers with $q>0$, then $q$ is a square. Further, if $q=4$, then $p \equiv 3(\bmod 4)$.

The proof is similar to that of Theorem 4. Moreover, a reasoning similar to that used to justify Example 5 gives rise to our second example family: 
Example 7. Consider the elliptic curve

$$
E: y^{2}+x y+y=x^{3}-\left(2+4 k+16 k^{2}+16 t\right) x^{2}+\left(12 k^{2}+3 k+8 t\right) x+\left(4 k^{2}+k+3 t\right),
$$

where $k, t \in \mathbb{Z}$. It has noninteger points $\left(\frac{3}{4}, \frac{1}{8}(-5+16 k)\right),\left(\frac{3}{4}, \frac{1}{8}(-9-16 k)\right)$ distinct from $\left(-\frac{1}{4},-\frac{3}{8}\right)$, so $E(\mathbb{Q})$ has rank at least 1 .

We now turn to the torsion groups of the curves $E_{1}$ and $E_{2}$. We know from Remark 3 that there is always a point of order 2; we wish to find conditions on the coefficients $a$ and $b$ that characterize when the torsion group is noncyclicwhich, by [Mazur 1977], means isomorphic to $\mathbb{Z} / 2 \mathbb{Z} \times \mathbb{Z} / 2 N \mathbb{Z}$, for $N=1,2,3,4$.

\section{Torsion subgroups on $E_{1}: y^{2}+x y=x^{3}+4(a-4 b) x^{2}+a x+b$}

Recall that $E_{1}$ has a torsion point of order 2 at $\left(-\frac{1}{4}, \frac{1}{8}\right)$ so long as it is nonsingular, a condition that boils down to $(a-4 b)^{2}-b \neq 0$, since the discriminant of $E_{1}$ factors as $(1-16 a+128 b)^{2}\left((a-4 b)^{2}-b\right)$, and the square factor is clearly nonzero.

Theorem 8. Consider the curve $E_{11}$ with equation (1-2), obtained as a particular case of $E_{1}$ with coefficients $a=4 m n+\frac{1}{2}(m+n)$ and $b=m n$, where $m$ and $n$ are integers satisfying $m \equiv n(\bmod 2)$ and $m<n$. The 2-torsion subgroup of $E_{11}$ is $E_{11, \text { tors }}(\mathbb{Q})[2]=\left\{O,\left(-\frac{1}{4}, \frac{1}{8}\right),(-2 m, m),(-2 n, n)\right\} \cong \mathbb{Z} / 2 \mathbb{Z} \times \mathbb{Z} / 2 \mathbb{Z}$.

All other curves $E_{1}$ not of this form have $E_{1, \text { tors }}(\mathbb{Q})[2]=\left\{O,\left(-\frac{1}{4}, \frac{1}{8}\right)\right\} \cong \mathbb{Z} / 2 \mathbb{Z}$.

Proof. Let $E_{1}$ have a torsion point $P$ of order 2 other than $\left(-\frac{1}{4}, \frac{1}{8}\right)$. From the proof of Theorem 1, and especially (2-3), we know that $P$ has the form $(q / 4,-q / 8)$ for $q \in \mathbb{Z}$. From the curve's equation we get $(1+q)\left(q^{2}+16(a-4 b) q+64 b\right)=0$. But $q \neq-1$ by assumption, so

$$
q=-8(a-4 b) \pm 8 \sqrt{(a-4 b)^{2}-b} .
$$

That is, $(a-4 b)^{2}-b=A^{2}$ for some integer $A$, which must be nonzero by the observation at the start of this section. Setting $B=a-4 b, m=B-A$ and $n=B+A$, so that $b=B^{2}-A^{2}=m n$ and $a=4 m n+(m+n) / 2$, we obtain the equation of $E_{11}$ in the theorem, with the side conditions on $m$ and $n$. (We know that $m \neq n$ since $A \neq 0$, and we can interchange $m$ and $n$ if necessary to ensure that $m<n$.)

This shows the last assertion of the theorem, and confirms that there cannot be more than three points of order 2, since there are only two choices of $q$ in (3-1). There remains to note that for any $m$ and $n$ as in the statement of the theorem (equivalently, for any integers $A, B$ with $A \neq 0$ ) we do indeed get torsion points of order 2 via (3-1), arising from $q=-8 m$ and $q=-8 n$.

We next recall a classical result; see for example [Knapp 1992, Theorem 4.2]. 
Lemma 9. Consider the elliptic curve

$$
E: y^{2}=\left(x-x_{1}\right)\left(x-x_{2}\right)\left(x-x_{3}\right) .
$$

For $(x, y) \in E(\mathbb{Q})$, there exists $P \in E(\mathbb{Q})$ with $2 P=(x, y)$ if and only if $x-x_{1}$, $x-x_{2}, x-x_{3}$ are squares.

Theorem 10. Let $k$ and $s$ be integers, with $s>0$. If the curve $E_{11}$ of Theorem 8 has coefficients $m=-k-2 k^{2}$ and $n=-k-2 k^{2}+2 s^{2}$, then it has a torsion subgroup isomorphic to $\mathbb{Z} / 2 \mathbb{Z} \times \mathbb{Z} / 4 \mathbb{Z}$.

Proof. The coordinate change $y=\eta-x / 2$ followed by $(x, \eta)=(X / 4, Y / 8)$ transforms $E_{11}$ into the curve

$$
E_{11}^{\prime}: Y^{2}=(X+1)(X+8 m)(X+8 n) .
$$

The points $\left(-\frac{1}{4}, \frac{1}{8}\right),(-2 m, m)$ and $(-2 n, n)$ of order 2 in $E_{11}(\mathbb{Q})$ correspond to $(-1,0),(-8 m, 0)$ and $(-8 n, 0)$ in $E_{11}^{\prime}(\mathbb{Q})$. We now ask which, if any, of these points can be the double of some point $P$ in $E_{11}^{\prime}(\mathbb{Q})$.

If $2 P=(-1,0)$, the differences $-1-(-1),-1-(-8 m)$ and $-1-(-8 n)$ must be squares, by Lemma 9 . But $8 m-1$ is certainly not a square, because it has residue $3(\bmod 4)$. Therefore $(-1,0)$ is not a double. Similarly, $(-8 n, 0)$ is not a double because $-8 n-(-8 m)<0$ is not a square. Finally $(-8 m, 0)$ is a double if and only if $-8 m-(-1)$ and $-8 m-(-8 n)$ are squares; that is, if and only if $8(n-m)=S^{2}$ and $1-8 m=K^{2}$, where $S, K$ are integers. Clearly $S \equiv 0(\bmod 4)$ and $K$ is odd; by interchanging $K$ and $-K$ we can ensure that $K \equiv 1(\bmod 4)$. So $E_{11}^{\prime}(\mathbb{Q})$, and hence also $E_{11}(\mathbb{Q})$, has torsion points of order 4 if and only if

$$
n-m=2 s^{2} \text { with } s \in \mathbb{Z}, s>0 \text { and } 1-8 m=(4 k+1)^{2} \text { with } k \in \mathbb{Z} \text {, }
$$

or equivalently if $m=-k-2 k^{2}$ and $n=-k-2 k^{2}+2 s^{2}$ for $k, s \in \mathbb{Z}$ with $s>0$.

Remark 11. Here is the explicit form of the points of order 4 in $E_{11}^{\prime}$ :

$$
\left\{\begin{array}{l}
P_{1}=\left(4\left(2 k+4 k^{2}-s(1+4 k)\right), \quad 4 s(1+4 k)(1+4 k-4 s)\right) \\
P_{2}=\left(4\left(2 k+4 k^{2}-s(1+4 k)\right),-4 s(1+4 k)(1+4 k-4 s)\right) \\
P_{3}=\left(4\left(2 k+4 k^{2}+s(1+4 k)\right), \quad 4 s(1+4 k)(1+4 k+4 s)\right) \\
P_{4}=\left(4\left(2 k+4 k^{2}+s(1+4 k)\right),-4 s(1+4 k)(1+4 k+4 s)\right)
\end{array}\right.
$$

We now recapitulate and complement our results for $E_{1}$, giving criteria for the occurrence of each torsion group $\mathbb{Z} / 2 \mathbb{Z} \times \mathbb{Z} / n \mathbb{Z}, n=1,2,3,4$.

Theorem 12. Consider the elliptic curve $E_{1}: y^{2}+x y=x^{3}+4(a-4 b) x^{2}+a x+b$, where $a, b \in \mathbb{Z}$. and recall that $\left(-\frac{1}{4}, \frac{1}{8}\right)$ is a point of order 2 in $E_{1}(\mathbb{Q})$.

(a) $E_{1}(\mathbb{Q})$ has a subgroup isomorphic to $\mathbb{Z} / 2 \mathbb{Z} \times \mathbb{Z} / 2 \mathbb{Z}$ if and only if the equation $t^{2}-2(a-4 b) t+b=0$ in $t$ has two integer solutions $m<n$, in which case $E_{1}$ has the form $E_{11}$ of (1-2): $y^{2}+x y=x^{3}+2(m+n) x^{2}+\left(4 m n+\frac{1}{2}(m+n)\right) x+m n$. 
(b) Assuming the condition in (a) is met, $E_{11}(\mathbb{Q})$ has a subgroup isomorphic to $\mathbb{Z} / 2 \mathbb{Z} \times \mathbb{Z} / 4 \mathbb{Z}$ if and only if $1-8 m$ and $\frac{1}{2}(n-m)$ are square integers.

(c) In the situation of (b), the full torsion group is $\mathbb{Z} / 2 \mathbb{Z} \times \mathbb{Z} / 8 \mathbb{Z}$ if and only if

$$
\sqrt{\frac{n-m}{2}}\left(4 \sqrt{\frac{n-m}{2}}+\sqrt{1-8 m}\right) \text { and } \sqrt{1-8 m}\left(4 \sqrt{\frac{n-m}{2}}+\sqrt{1-8 m}\right)
$$

are square integers.

(d) Assuming the condition in (a) is met, the full torsion group is $\mathbb{Z} / 2 \mathbb{Z} \times \mathbb{Z} / 6 \mathbb{Z}$ if and only if there exist integers $\alpha, \beta$ such that $|\alpha|<\beta, \gamma:=-\alpha \beta /(\alpha+\beta)$ is an integer, $m=\frac{1}{8}\left(\alpha^{2}-\gamma^{2}+1\right)$, and $n=\frac{1}{8}\left(\beta^{2}-\gamma^{2}+1\right)$. In this situation the points of order 3 have $x$-coordinate $\frac{1}{4}\left(\gamma^{2}-1\right)$; moreover $\gamma$ is odd and $\beta \equiv \alpha \equiv 0$ $(\bmod 4)$.

Proof. Part (a) is just a restatement of Theorem 8, apart from the easily checked equivalence between the conditions $a=4 m n+\frac{1}{2}(m+n)$ and $b=m n$ in that theorem and $m, n$ being the roots of the quadratic equation $t^{2}-2(a-4 b) t+b=0$.

(b) If $1-8 m$ and $(n-m) / 2$ are squares, the quantities $s=\sqrt{(n-m) / 2}$ and $k=$ $(-1+\sqrt{1-8 m}) / 4$ or $k=(-1-\sqrt{1-8 m}) / 4$ satisfy the conditions of Theorem 10 . (We choose whichever definition of $k$ yields an integer; note that $\sqrt{1-8 m}$ is odd.) Therefore in this situation $E_{11}$ has a torsion subgroup isomorphic to $\mathbb{Z} / 2 \mathbb{Z} \times \mathbb{Z} / 4 \mathbb{Z}$. Conversely, if $E_{11}$ has a torsion subgroup isomorphic to $\mathbb{Z} / 2 \mathbb{Z} \times \mathbb{Z} / 4 \mathbb{Z}$, we have $m=-k-2 k^{2}$ and $n=-k-2 k^{2}+2 s^{2}$ for some integers $k, s$ with $s>0$.

(c) Let $s$ and $k$ be as above, and recall the short form $E_{11}^{\prime}$ of the curve given in (3-3). We ask when the points $P_{1}, P_{2}, P_{3}, P_{4}$ of order four listed in (3-4) are doubles. Consider first the condition imposed by Lemma 9 for $P_{1}$ to be a double. It is that the following three differences be squares:

$$
\begin{aligned}
& 4\left(2 k+4 k^{2}-s(1+4 k)\right)+1=(1+4 k)(1+4 k-4 s), \\
& 4\left(2 k+4 k^{2}-s(1+4 k)\right)+8 m=-4 s(1+4 k), \\
& 4\left(2 k+4 k^{2}-s(1+4 k)\right)+8 n=-4 s(1+4 k-4 s) .
\end{aligned}
$$

Clearly if any two are squares, so is the third. We discard the middle line and rewrite the other two right-hand sides in terms of $m$ and $n$, using the expressions in (b). We must take the minus sign in $k=(-1 \pm \sqrt{1-8 m}) / 4$, since $s$ is positive and $-4 s(1+4 k)$ is a square. It follows that the condition for $P_{1}$ to be a double is that the quantities in (3-5) be square integers. The same holds for $P_{2}$, since it has the same $x$-coordinate as $P_{1}$.

A similar argument shows that the condition on the integers $s>0$ and $k$ for $P_{3}$ (or $\left.P_{4}\right)$ to be a double is that $s(1+4 k+4 s)$ and $(1+4 k)(1+4 k+4 s)$ be squares. Substituting $k=(-1+\sqrt{1-8 m}) / 4$ and $s$ leads to the same expressions (3-5) in terms of $m$ and $n$. Thus there is a point of order 8 in the curve if and only if 
both quantities in (3-5) are square integers. (Because of the different relationship between $k$ and $m$ in each case, $P_{1}$ and $P_{2}$ being doubles is mutually exclusive with $P_{3}$ and $P_{4}$ being doubles, as expected from the structure of $\mathbb{Z} / 2 \mathbb{Z} \times \mathbb{Z} / 8 \mathbb{Z}$.)

(d) Assume the curve has a point of order 3, and let $\left(X_{P}, Y_{P}\right)$ be its coordinates in the alternate equation (3-3) introduced in the proof of Theorem 10. Because this point is the double of a generator of $\mathbb{Z} / 6 \mathbb{Z}$, we can apply Lemma 9 to conclude that $A:=X_{P}+8 m, B:=X_{P}+8 n, G:=X_{P}+1$ are all square integers.

Meanwhile, the standard algebraic constraint for a point on an elliptic curve of the form (3-2) to have order three (easy to derive using the characterization of such a point as an inflection point) amounts in this case to

$$
4 A B G(A+B+G)-(B G+A B+A G)^{2}=0 .
$$

If we let $\alpha, \beta, \gamma$ be the nonnegative square roots of $A, B, G$ (note that $\alpha<\beta$ since $m<n)$, we can write the left-hand side of (3-6) as

$$
(\alpha \beta+\beta \gamma+\alpha \gamma)(-\alpha \beta+\beta \gamma+\alpha \gamma)(\alpha \beta-\beta \gamma+\alpha \gamma)(\alpha \beta+\beta \gamma-\alpha \gamma),
$$

so one of these factors vanishes. By changing the sign of $\alpha$ and/or $\gamma$ we can ensure that $\alpha \beta+\beta \gamma+\alpha \gamma=0$, while $\beta$ remains positive and greater than $|\alpha|$. Thus $\gamma=-\alpha \beta /(\alpha+\beta)$. Substitution also gives, successively,

$$
X_{P}=\gamma^{2}-1, \quad m=\frac{1}{8}\left(\alpha^{2}-\gamma^{2}+1\right), \quad n=\frac{1}{8}\left(\beta^{2}-\gamma^{2}+1\right) .
$$

Recalling that the $x$-coordinate in $E_{11}$ is related to the $X$-coordinate in $E_{11}^{\prime}$ by $x=X / 4$, we deduce that $x=\frac{1}{4}\left(\gamma^{2}-1\right)$. The divisibility conditions on $\gamma, \alpha, \beta$ follow since $x, m, n$ are integers. This concludes one direction of the proof.

The other direction is a matter of checking (using the same algebra) that, given integers $\alpha, \beta, \gamma$ with $\gamma=-\alpha \beta /(\alpha+\beta), m=\frac{1}{8}\left(\alpha^{2}-\gamma^{2}+1\right)$, and $n=\frac{1}{8}\left(\beta^{2}-\gamma^{2}+1\right)$, the points on $E_{11}$ with $x=\frac{1}{4}\left(\gamma^{2}-1\right)$ have order 3 .

\section{Torsion subgroups on $E_{2}: y^{2}+x y+y=x^{3}+2(2 a-8 b-1) x^{2}+a x+b$}

Recall that $E_{2}$ has a torsion point of order 2 at $\left(-\frac{1}{4},-\frac{3}{8}\right)$ so long as it is nonsingular, a condition equivalent to $(a-4 b)^{2}-a+3 b \neq 0$, since the discriminant of $E_{2}$ factors as $(25-16 a+128 b)^{2}\left((a-4 b)^{2}-a+3 b\right)$, and the square factor is clearly nonzero.

\section{Theorem 13. Consider the curve}

$$
E_{22}: y^{2}+x y+y=x^{3}+2(m+n) x^{2}+\left(4 m n+\frac{1}{2}(m+n-1)\right) x+m n-\frac{1}{4}
$$

obtained as a particular case of $E_{2}$ with coefficients $a=4 m n+\frac{1}{2}(m+n)-\frac{1}{2}$ and $b=m n-\frac{1}{4}$, where $m$ and $n$ are half-integers (that is, $2 m, 2 n$ are odd integers) 
satisfying $m-n \equiv 0(\bmod 2)$ and $m<n$. Then $E_{22}$ does not have torsion points of order 4 , and the 2-torsion subgroup of $E_{22}$ is

$$
E_{22, \text { tors }}(\mathbb{Q})[2]=\left\{O,\left(-\frac{1}{4},-\frac{3}{8}\right),\left(-2 m, m-\frac{1}{2}\right),\left(-2 n, n-\frac{1}{2}\right)\right\} \cong \mathbb{Z} / 2 \mathbb{Z} \times \mathbb{Z} / 2 \mathbb{Z} .
$$

All other curves $E_{2}$ not of this form have $E_{2, \text { tors }}(\mathbb{Q})[2]=\left\{O,\left(-\frac{1}{4},-\frac{3}{8}\right)\right\} \cong \mathbb{Z} / 2 \mathbb{Z}$.

Proof. Assume that $E_{2}$ has a torsion point $P$ of order 2 other than $\left(-\frac{1}{4},-\frac{3}{8}\right)$, and write $P=\left(q / 4,-q / 8-\frac{1}{2}\right)$ with $q \in \mathbb{Z}$. From the equation of $E_{2}$ we obtain $(1+q)\left(q^{2}+8 q(2 a-8 b-1)+16(4 b+1)\right)=0$, so

$$
q=-4(2 a-8 b-1) \pm 4 \sqrt{(2 a-8 b-1)^{2}-(4 b+1)} .
$$

That is, $(2 a-8 b-1)^{2}-(4 b+1)=A^{2}$ for some even integer $A$, which must be nonzero by the observation at the start of this section (note that the radicand in (4-1) equals $\left.(a-4 b)^{2}-a+3 b\right)$. Setting $B=2 a-8 b-1, m=\frac{1}{2}(B+A)$ and $n=\frac{1}{2}(B-A)$, so that $b=\frac{1}{4}\left(B^{2}-A^{2}-1\right)=m n-\frac{1}{4}$ and $a=\frac{1}{2}(B+8 b+1)=\frac{1}{2}(m+n)+(4 m n-1)+\frac{1}{2}$, we obtain the equation of $E_{22}$ in the theorem, with the side conditions on $m$ and $n$.

This shows the last assertion of the theorem, and also that there cannot be more than three points of order 2, since there are only two choices of $q$ in (4-1). Further, for any $m$ and $n$ as in the statement of the theorem (equivalently, for any nonzero even integer $A$ and any odd integer $B$ ) we do indeed get torsion points of order 2 via (4-1), arising from $q=-8 m$ and $q=-8 n$.

There remains to show that $E_{22}(\mathbb{Q})$ has no torsion of order 4. To do this, apply the coordinate change $y=\eta-(x+1) / 2$ followed by $(x, \eta)=(X / 4, Y / 8)$. This transforms $E_{22}$ into the curve

$$
E_{22}^{\prime}: Y^{2}=(X+1)(X+8 m)(X+8 n),
$$

which is the same as $E_{11}^{\prime}$ of (3-3). The points of order two listed above map become $(-1,0),(-8 m, 0)$, and $(-8 n, 0)$. We then proceed as in the proof of Theorem 10 , with the difference that here $m, n$ are half-integers. First, $(-1,0)$ is not a double in $E_{22}^{\prime}(\mathbb{Q})$ because $-1-(-8 m) \equiv 3(\bmod 8)$ is not a square. Nor can $(-8 m, 0)$ be a double, since $-8 m-(-1) \equiv 5(\bmod 8)$. Similarly, $(-8 n, 0)$ cannot be a double.

Theorem 14. Consider the elliptic curve $E_{2}: y^{2}+x y+y=x^{3}+2(2 a-8 b-1) x^{2}+$ $a x+b$, where $a, b \in \mathbb{Z}$. and recall that $\left(-\frac{1}{4},-\frac{3}{8}\right)$ is a point of order 2 in $E_{2}(\mathbb{Q})$.

(a) If the equation $t^{2}-(2 a-8 b-1) t+b+\frac{1}{4}=0$ in $t$ has two distinct half-integer solutions $m<n$, then $E_{2}$ can be written as

$E_{22}: y^{2}+x y+y=x^{3}+2(m+n) x^{2}+\left(4 m n+\frac{1}{2}(m+n-1)\right) x+m n-\frac{1}{4}$

and $E_{2}(\mathbb{Q})$ has a subgroup isomorphic to $\mathbb{Z} / 2 \mathbb{Z} \times \mathbb{Z} / 2 \mathbb{Z}$.

(b) Never does $E_{2}(\mathbb{Q})$ have a subgroup isomorphic to $\mathbb{Z} / 2 \mathbb{Z} \times \mathbb{Z} / 4 \mathbb{Z}$. 
(c) Assuming the condition in (a) is met, the full torsion group is $\mathbb{Z} / 2 \mathbb{Z} \times \mathbb{Z} / 6 \mathbb{Z}$ if and only if there exist integers $\alpha, \beta$ such that $|\alpha|<\beta, \gamma:=-\alpha \beta /(\alpha+\beta)$ is an integer, $m=\frac{1}{8}\left(\alpha^{2}-\gamma^{2}+1\right)$, and $n=\frac{1}{8}\left(\beta^{2}-\gamma^{2}+1\right)$. In this situation the points of order 3 have $x$-coordinate $\frac{1}{4}\left(\gamma^{2}-1\right)$; moreover $\gamma$ is odd and $\beta \equiv \alpha \equiv 2$ $(\bmod 4)$.

Proof. Parts (a) and (b) restate Theorem 13, apart from the easily checked equivalence between the conditions $a=4 m n+\frac{1}{2}(m+n-1)$ and $b=m n-\frac{1}{4}$ in that theorem and $m, n$ being the roots of the quadratic equation $t^{2}-(2 a-8 b-1) t+b+\frac{1}{4}=0$.

The proof of part (c) is verbatim the same as that of Theorem 12(d). (The condition $m, n \in \mathbb{Z}$ was not used in that proof except to show that $\alpha, \beta$ were divisible by 4 . Here the condition $m, n \in \frac{1}{2} \mathbb{Z} \backslash \mathbb{Z}$ gives $\beta \equiv \alpha \equiv 2(\bmod 4)$ instead.)

\section{Characterization of curves with torsion subgroup $\mathbb{Z} / 2 \mathbb{Z} \times \mathbb{Z} / 8 \mathbb{Z}$ in terms of Pythagorean triples}

We now give a family of curves $E_{11}$ whose torsion subgroup is isomorphic to $\mathbb{Z} / 2 \mathbb{Z} \times \mathbb{Z} / 8 \mathbb{Z}$, and show that it is exhaustive.

Theorem 15. Let $t$ be a nonnegative integer and $(v, w, z)$ a primitive Pythagorean triple with $v$ even. For the integers

$$
s=\frac{v^{2}(4 t+1)}{4}, k=\frac{w^{2}(4 t+1)-1}{4} \text { or } s=\frac{v^{2}(4 t+3)}{4}, k=-\frac{w^{2}(4 t+3)+1}{4},
$$

the conditions in part (c) of Theorem 12 are satisfied, so the elliptic curve $E_{11}$ written there has a torsion subgroup isomorphic to $\mathbb{Z} / 2 \mathbb{Z} \times \mathbb{Z} / 8 \mathbb{Z}$.

Conversely, if $E_{11}$ in Theorem 12 has a torsion point of order 8, we are in the situation of part (c) of that theorem, with $s$ and $k$ arising from a primitive Pythagorean triple as above.

Proof. Recall from the proof of Theorem 12(c) that the condition for the existence of torsion of order 8 is that there should be integers $B, C$ satisfying either

$$
B^{2}=s(1+4 k+4 s) \quad \text { and } \quad C^{2}=(1+4 k)(1+4 k+4 s)
$$

or

$$
B^{2}=-s(1+4 k-4 s) \quad \text { and } \quad C^{2}=(1+4 k)(1+4 k-4 s) .
$$

Substituting $s=\frac{1}{4} v^{2}(4 t+1)$ and $k=\frac{1}{4}\left(w^{2}(4 t+1)-1\right)$ on the left-hand sides of equalities (5-1) leads to

$$
\frac{1}{4} v^{2}(4 t+1)^{2}\left(v^{2}+w^{2}\right), \quad w^{2}(4 t+1)^{2}\left(v^{2}+w^{2}\right),
$$

which are squares because $v$ is even and $v^{2}+w^{2}=z^{2}$. Similarly, substituting $s=\frac{1}{4} v^{2}(4 t+3)$ and $k=-\frac{1}{4}\left(w^{2}(4 t+3)+1\right)$ in (5-2) also yields squares. 
Conversely, suppose (5-1) is satisfied; our job is to find a Pythagorean triple as in the statement of Theorem 15. Combining the two equations (5-1) we get $4 B^{2}+C^{2}=(1+4 k+4 s)^{2}$, so

$$
(2 B, C, 1+4 k+4 s)
$$

is a Pythagorean triple. Let $c$ be the gcd of the three members and $(v, w, z)$ the corresponding primitive triple, so $2 B=c v, C=c w, 1+4 k+4 s=c z$. This last equation shows that $c$ is odd, so $v$ is even, so $w$ is odd. Now note that

$$
4 s(1+4 k+4 s)=c^{2} v^{2}, \quad(1+4 k)(1+4 k+4 s)=c^{2} w^{2} .
$$

Since $c z=1+4 k+4 s$ divides both $c^{2} v^{2}$ and $c^{2} w^{2}$, and since $v, w, z$ are relatively prime, we conclude that $z$ divides $c$, that is, $c=z u$ for some odd integer $u$. Hence

$$
4 s=v^{2} u, \quad 1+4 k=w^{2} u .
$$

This last equation gives $u \equiv 1(\bmod 4)$ since $w^{2} \equiv 1(\bmod 4)$. Thus we can write $u=4 t+1$ for some integer $t \geq 0$, and from (5-3) we get

$$
s=\frac{v^{2}(4 t+1)}{4} \quad \text { and } \quad k=\frac{w^{2}(4 t+1)-1}{4},
$$

as needed.

A wholly analogous reasoning shows that when (5-2) is satisfied, instead of (5-1), there is a Pythagorean triple $(v, w, z)$ (with $v$ even) and an integer $t \geq 0$ such that

$$
s=\frac{v^{2}(4 t+3)}{4} \quad \text { and } \quad k=-\frac{w^{2}(4 t+3)+1}{4} .
$$

Remark 16. Every elliptic curve over $\mathbb{Q}$ with torsion group $\mathbb{Z} / 2 \mathbb{Z} \times \mathbb{Z} / 8 \mathbb{Z}$ is isomorphic to one of those in Theorem 15. Indeed, it is known (see [Campbell and Goins 2004, Theorem 6.2], for example) that every such curve has an equation of the form

$$
y^{2}=x\left(x+u^{2}\right)\left(x+u^{-2}\right), \quad \text { for } u=\frac{T^{2}-1}{2 T} \text { with } T \in \mathbb{Q} \backslash\{0,1,-1\} .
$$

It's easy to see that there is a primitive Pythagorean triple $(v, w, z)$ with $v / w=u$, and by interchanging $v$ and $w$ if necessary we can ensure that $v$ is even. To go from the form

$$
y^{2}=x\left(x+\frac{w^{2}}{v^{2}}\right)\left(x+\frac{v^{2}}{w^{2}}\right)
$$

to the desired form of $E_{11}$, we apply affine coordinate changes with rational coefficients: the scaling $(x, y) \mapsto\left(4 x v^{-2} w^{-2}, 8 y v^{-3} w^{-3}\right)$, followed by the change of 
parameters $s=\frac{1}{4} v^{2}$ and $k=\frac{1}{4}\left(w^{2}-1\right)$ (case $t=0$ in the first set of substitution in Theorem 15), gives

$$
y^{2}=x\left(x+(2 s)^{2}\right)\left(x+\left(2 k+\frac{1}{2}\right)^{2}\right) ;
$$

further inserting the values $m=-k-2 k^{2}$ and $n=-k-2 k^{2}+2 s^{2}$ and applying the coordinate change $x \mapsto x+2 m$ followed by $y \mapsto y+x / 2$ leads to the canonical form of $E_{11}$.

\section{References}

[Campbell and Goins 2004] G. Campbell and E. H. Goins, "Heron triangles, Diophantine problems and elliptic curves", preprint, 2004, Available at http://www.swarthmore.edu/NatSci/gcampbe1/ papers/heron-Campbell-Goins.pdf.

[Knapp 1992] A. W. Knapp, "Elliptic curves", 1992. MR 93j:11032 Zbl 0804.14013

[Mazur 1977] B. Mazur, "Modular curves and the Eisenstein ideal", Inst. Hautes Études Sci. Publ. Math. 47 (1977), 33-186. MR 80c:14015 Zbl 0394.14008

Received October 26, 2007. Revised September 2, 2008.

SUMI JEONG

Department of Mathematics and Institute of Pure and Applied Mathematics

CHONBUK NATIONAL UNIVERSITY

JEONJU, JEONBUK 561-756

REPUBLIC OF KOREA

sumi@chonbuk.ac.kr

HWASIN PARK

Department of Mathematics and Institute of Pure and Applied Mathematics CHONBUK NATIONAL UNIVERSITY

JEONJU, JEONBUK 561-756

REPUBLIC OF KOREA

park@chonbuk.ac.kr 